\title{
Comparative Analysis of Flame Characteristics of Castor Oil and Some Other Inorganic Flame Retardants Used in Polyurethane Foam Systems
}

\author{
Polycarp .0. Ikeh \\ Department of Pure and Applied Chemistry, Usmanu Danfodiyo University, Sokoto, Nigeria. \\ [E-mail: poikeh@yahoo.co.uk; Tel.: +234(0)8035950598]
}

\begin{abstract}
The flame characteristics of castor oil based foam and that of polyether foam impregnated with inorganic flame retardants (FR) were investigated. The polyether foams were impregnated with measured concentration of Antimony trioxide and Sodium bromide, Ammonium dihydrogen orthophosphate, Diammonium hydrogen orthophosphate, and Castor oil containing zero flame retardant. Incorporating these flame retardants into polyurethane formulations gave variable flame characteristics such as ignition time, flame propagation rate, after glow, char rate, add-on and glow time. These properties were measured as a function of concentration and type of flame retardant incorporated into the formulation. Result showed that ignition time and Add-On showed significant increase in this sequenceCastor oil > Ammonium dihydrogen orthophosphate> Diammonium hydrogen orthophosphate>Antimony trioxide/Sodium bromide, at all the different concentrations, while flame-propagation rate and After-glow decreased in the same sequence for all the FR used at different concentrations. However the \%Char increased in a reverse order $\ddot{i}$ Diammonium hydrogen orthophosphate>Ammonium dihydrogen orthophosphate>Antimony trioxide/Sodium bromide>Castor oil. These results show that castor oil is an effective flame retardant compared to the inorganic flame-retardants.
\end{abstract}

Keywords: Flame retardants; Polyurethane; Ignition time; Form; Add-on

\section{INTRODUCTION}

Most of the immediate surroundings of man are combustible-clothes; furniture, construction materials, interior decorations and the commonest interior decorations are foamed plastics. It is a common knowledge that foamable materials burns vigorously and ignite readily under the right conditions.

Humans have always been plagued by unwanted fire and properties worth millions of naira have been gutted by fire. Therefore the need to seek for efficient and affordable ways of reducing the combustibility of our surroundings is of primary importance. Foamed polyurethane are made of organic constituents which are themselves flammable and volatile and incorporation of a fire retardant chemical to a flammable polymer system will reduce the combustion rate of such polymer (Calamari and Harper,1993) Polyurethane foams have highly cellular structures, which are easily ignitable and highly flammable. Flame spreads very fast on the surface of these materials and results in engulfing the entire area in a few minutes.
The burning process of polyurethane foam maybe considered as occurring in five stages

(i) Heat from external source is applied to foam, and it progressively raises the temperature. The thermal insulation properties of the foam promote a rapid temperature rise at the exposed surface because heat cannot be transmitted to the lower layer of the foam.

(ii) The polyurethane foam reaches its temperature of initial decomposition and begins to form combustible gases, noncombustible gases, entrained solid particles and carbonaceous char. The evolution of gases expands the foam structure and thus combustion and non combustible gases will both cause difficulty by disrupting the chemical and physical structure of the foam, exposing new surfaces to destructive temperatures.

(iii) The resulting combustible gases ignite in the presence of sufficient oxygen and further combustion begins. The condition of ignition depends on the presence of an external source of ignition and the 
temperature and composition of the gas phase.

(iv) The heat of combustion raises the temperature of the gaseous products of combustion and of the non combustible gases resulting in an increased heat transfer by conduction.

(v) The heat will transfer from the combustible zone to adjacent foam surface producing further decomposition and ignition thus flame propagation.

Polyurethane flame retardation requires, cheap, reactive and miscible chemicals that can bind easily with the foam formulation to reduce flame propagation rate without adversely affecting the foam density.

It is a common practice that because of cost and profit driven attitude of most foam manufacturers, they avoid the use of these chemicals and opt for inert fillers (sawdust, sodium carbonate, fibers etc). These inert fillers are use because of the inherent advantage of enhancing solidity and volume expansion at the expense of combustibility (Boehling and Cooper, 1985). In many polymers the high concentration of halogenated organic compounds needed to impart flame retardancy adversely affects their physical properties. In practice halogen containing flame-retardants are formulated with inorganic compounds that behave synergistically with the halogens.

In this work effort was made to compare the flame retardant properties of antimony trioxide and organophosphorous compounds with unsaturated polyester (castor oil) in polyurethane foams.

\section{EXPERIMENTAL}

Materials: Antimony trioxide and Sodium bromide, diammonium hydrogen orthophosphate and ammonium dihydrogen orthophosphate (BDH) were obtained from the chemistry laboratory. Castor oil was extracted from the raw seeds (obtained from the Sokoto market). The following materials viz: stannous octoeate and dimethylethanolamine (Goldsmith. AG), methylene chloride (Solitary), toluene diisocyanate (TDI, 80:20 of 2,4 and 2,6 isomers respectively) (Enchain), Silicone oil and Polyol (Liberty chemicals, London) and Distil water (chemistry laboratory) were kindly supplied by Concord Foam Nigeria Plc, Sokoto.

\section{Methods}

Foam Preparation and Flame Retardant Treatment: Variable formulations of foam recipes were prepared using the formulation in Table 1. Polyol, water, silicone oil, stannous octoate, dimethylethanolamine and the flameretardants were thoroughly mixed together in a mould. Afterwards, toluene diisocyanate was added to the mixture, with continued stirring until properly blended. The mixture was stirred with an electric stirrer to ensure good dispersion of reagents and foam of desirable cell structure. The stirring continued until foaming commenced, before stopping to allow the mixture to form. The foam samples produced were left to stand for 48 hours before the flame test to ensure complete curing.FRA-Foam incorporated with Antimony trioxide and Sodium bromide, FRB-Foam incorporated with Ammonium dihydrogen orthophosphate,FRC- Foam incorporated with Diammonium hydrogen orthophosphate and FRDFoam incorporated with castor oil (Herington and Hock, 1991).

\section{Flame Properties}

Add-On \%: -Flexible polyurethane foams were prepared using variable amount of flame retardants added insitu and blank formulation of foams without flame retardants. Rectangle shaped foam materials were cut from treated and untreated samples with known dimensions. The weight of the foam with flame retardant $\left(\mathrm{W}_{\mathrm{a}}\right)$ minus the weight of foam without flame retardant $\left(\mathrm{W}_{\mathrm{b}}\right)$ divided by the weight of foam without flame retardant multiplied by 100 (Garba and Eboatu, 1990).

$$
\text { Add - On }(\%)=\frac{\mathrm{W}_{\mathrm{a}}-\mathrm{W}_{\mathrm{b}}}{\mathrm{W}_{\mathrm{b}}} \times 100
$$

Ignition Time: The samples were clamped vertically at a distance of $5.00 \mathrm{~cm}$ from the surface of a gas burner, which was adjusted to give steady flame. The time taken by the sample to catch fire was recorded as the ignition time (Garba and Eboatu, 1990). 
Flame Propagation Rate (FP): The sample was clamped vertically and then ignition at the base in a draught-free room. The distance traveled at a stipulated time interval by the char front was measured and the rate of flame propagation was calculated as the vertical distance traversed per second (Garba and Eboatu, 1990).

Afterglow Time: This was measured by recording the time between flame out and the last visually perceptible glow (Garba and Eboatu, 1990).
Char Formation: The sample was crushed into pieces and weighed, after which it was kept in a crucible with a lid. This was then placed into an electrically heated muffle furnace at $700^{\circ} \mathrm{C}$.The crucible with its contents were periodically cooled and weighed at 20mins interval until constant weight was obtained. The amount of char formed was determined using the expression (Garba and Eboatu, 1990).

Char $(\%)=\frac{\text { Weight of material after burning }}{\text { Weight of material before burning }} \times 100$

Table 1: Formulation for the preparation of polyurethane foam samples

\begin{tabular}{|c|c|c|c|c|c|c|c|c|c|c|}
\hline \multirow[b]{2}{*}{ Samples } & \multicolumn{10}{|c|}{ Reagents (pbw) } \\
\hline & Polyol & $\begin{array}{c}\text { Silicone } \\
\text { oil }\end{array}$ & $\begin{array}{l}\text { Stannous } \\
\text { octoate }\end{array}$ & DMEA & Water & TDI & $\begin{array}{l}\text { FRA/ } \\
\mathrm{NaBr}\end{array}$ & FRB & FRC & FRD \\
\hline Blank & 12.0 & 0.2 & 0.1 & 0.1 & 0.5 & 2.5 & 0.0 & 0.0 & 0.0 & 0.0 \\
\hline FRA1 & 12.0 & 0.2 & 0.1 & 0.1 & 0.5 & 2.5 & 0.5 & 0.0 & 0.0 & 0.0 \\
\hline FRA2 & 12.0 & 0.2 & 0.1 & 0.1 & 0.5 & 2.5 & 1.0 & 0.0 & 0.0 & 0.0 \\
\hline FRA3 & 12.0 & 0.2 & 0.1 & 0.1 & 0.5 & 2.5 & 1.5 & 0.0 & 0.0 & 0.0 \\
\hline FRA4 & 12.0 & 0.2 & 0.1 & 0.1 & 0.5 & 2.5 & 2.0 & 0.0 & 0.0 & 0.0 \\
\hline FRA5 & 12.0 & 0.2 & 0.1 & 0.1 & 0.5 & 2.5 & 2.5 & 0.0 & 0.0 & 0.0 \\
\hline FRB1 & 12.0 & 0.2 & 0.1 & 0.1 & 0.5 & 2.5 & 0.0 & 0.5 & 0.0 & 0.0 \\
\hline FRB2 & 12.0 & 0.2 & 0.1 & 0.1 & 0.5 & 2.5 & 0.0 & 1.0 & 0.0 & 0.0 \\
\hline FRB3 & 12.0 & 0.2 & 0.1 & 0.1 & 0.5 & 2.5 & 0.0 & 1.5 & 0.0 & 0.0 \\
\hline FRB4 & 12.0 & 0.2 & 0.1 & 0.1 & 0.5 & 2.5 & 0.0 & 2.0 & 0.0 & 0.0 \\
\hline FRB5 & 12.0 & 0.2 & 0.1 & 0.1 & 0.5 & 2.5 & 0.0 & 2.5 & 0.0 & 0.0 \\
\hline FRC1 & 12.0 & 0.2 & 0.1 & 0.1 & 0.5 & 2.5 & 0.0 & 0.0 & 0.5 & 0.0 \\
\hline FRC2 & 12.0 & 0.2 & 0.1 & 0.1 & 0.5 & 2.5 & 0.0 & 0.0 & 1.0 & 0.0 \\
\hline FRC3 & 12.0 & 0.2 & 0.1 & 0.1 & 0.5 & 2.5 & 0.0 & 0.0 & 1.5 & 0.0 \\
\hline FRC4 & 12.0 & 0.2 & 0.1 & 0.1 & 0.5 & 2.5 & 0.0 & 0.0 & 2.0 & 0.0 \\
\hline FRC5 & 12.0 & 0.2 & 0.1 & 0.1 & 0.5 & 2.5 & 0.0 & 0.0 & 2.5 & 0.0 \\
\hline FRD1 & 12.0 & 0.2 & 0.1 & 0.1 & 0.5 & 2.5 & 0.0 & 0.0 & 0.0 & 0.5 \\
\hline FRD2 & 12.0 & 0.2 & 0.1 & 0.1 & 0.5 & 2.5 & 0.0 & 0.0 & 0.0 & 1.0 \\
\hline FRD3 & 12.0 & 0.2 & 0.1 & 0.1 & 0.5 & 2.5 & 0.0 & 0.0 & 0.0 & 1.5 \\
\hline FRD4 & 12.0 & 0.2 & 0.1 & 0.1 & 0.5 & 2.5 & 0.0 & 0.0 & 0.0 & 2.0 \\
\hline FRD5 & 12.0 & 0.2 & 0.1 & 0.1 & 0.5 & 2.5 & 0.0 & 0.0 & 0.0 & 2.5 \\
\hline
\end{tabular}

$\mathrm{FRA}=$ Foam incorporated with Antimony trioxide/Sodium bromide

$\mathrm{FRB}=$ Foam incorporated with Ammonium dihydrogen orthophosphate

$\mathrm{FRC}=$ Foam incorporated with Diammonium hydrogen orthophosphate

$\mathrm{FRD}=$ Foam incorporated with Castor oil

\section{RESULTS AND DISCUSSIONS Results}

Results shown in Figs 1-5 for polyurethane foam impregnated with the different flame-retardants depicts the comparative picture of the effects of these flame-retardants on the flame properties of polyurethane foams. Fig. 1 shows the relationship between the flame retardant and the ignition time, for polyurethane foam containing different amount and types of flame-retardants. The results showed that the ignition time increases with increase in FR concentration, for all the formulations, beyond which no ignition occurred, and rate of increase depended on the type of FR 
used. A glance at the graph showed that the castor oil gave higher ignition time compared to the inorganic FR.This dependence of ignition time on the concentration and type of FR has been interpreted in terms of two sequential controlling mechanisms in the spontaneous ignition processes (Gann, 1993).

(i) Heating the material up to its decomposition temperature; and

(ii) Decomposition of the material to produce a mixture of combustible gases which will diffuse and ignite if its concentration lies within the flammability limits.

In Figure 2, the flame propagation rate decreases with increase in FR concentration in all the cases. The rapid decrease in flame propagation rate of the samples with different types and concentration of FR can be explained by the fact that the antimony trioxide is a synergistic co-additive, in combination with halogen compounds $(\mathrm{NaBr})$ facilitating the reduction in total flame retardant levels needed to achieve a desired level of flame retardancy. This is usually accomplished by release of halogen acids at fire temperatures. The halogen acids react with the antimony $і$ containing materials to form antimony trihalide and/orantimony halide oxide. These materials act both in the foam and in the flame to suppress flame propagation in the condensed phase. They promote char formation, which acts as a physical barrier to flame and inhibits the volatilization of flammable materials (Khattab et al., 1992). In the flame, antimony halides and halide oxides, generate in sufficient volume, provide an inert gas blanket over the substrate, thus excluding oxygen and preventing flame spread. The phosphorous compounds act by the formation of a carbonaceous layer in the condensed phase and fragments that interrupt the radical chain mechanism. Castor oil increases foam density and rigidity thus increase the bond strength of the foam. This offers a serious resistance to bond breaking process.

The Add-On increased generally in Figure 3 and this indicated that Add-On increases due to the porosity index and density of the foam (Garba and Eboatu, 1990). This is expected considering the fact that the indices increased from antimony trioxide to castor oil. The antimony is halogenated by the addition of sodium bromide and the presence of the halogen reduces the vapor pressure and water solubility of the flame retardant, thereby contributing to the retention of the flame retardant in the polymer.

In Figures 4 and 5 the After-glow and the char decreased in this order-antimony trioxide/sodium bromide> Diammonium hydrogen orthophosphate $>$ ammonium dihydrogenorthophosphate > castor oil with increase in concentration and type or FR.The use of antimony trioxide and bromine simultaneously in polyurethane is effective since in a burning process the major part of the antimony is volatized, probably as $\mathrm{SbBr}_{3}$ or $\mathrm{SbOBr}$. These compounds then provide a ready source of hydrogen bromide and they also produce in the middle of the combustion zone a mist of fine particles of solid $\mathrm{SbO}$, which can catalise the recombination of the free radicals responsible for flame propagation, via the formation of transient species like $\mathrm{SbOH}$ (Cullis, 1987) 


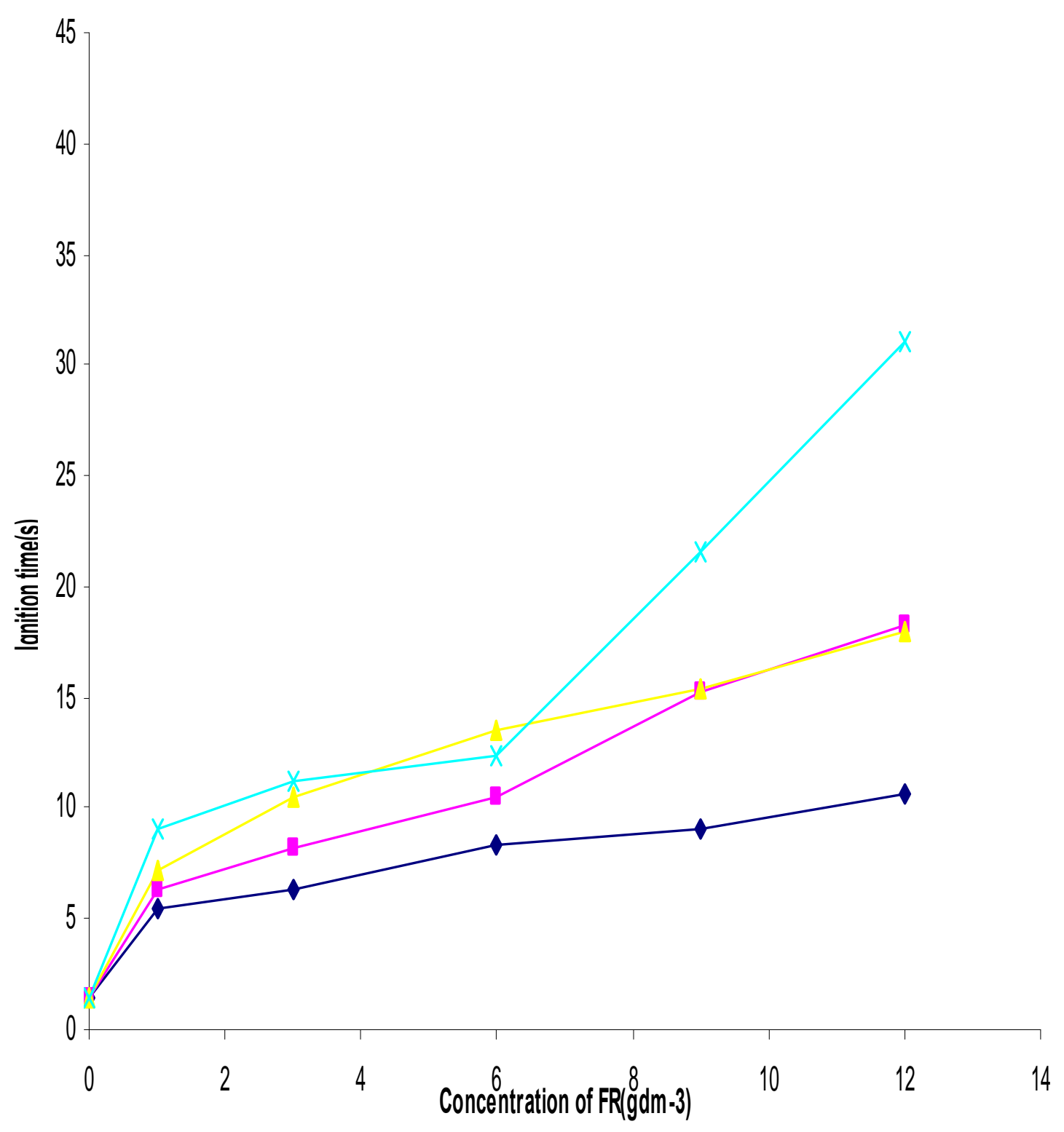

Figure 1.Plot of ignition time of polyurethane foam versus $F R$ concentration

$\rightarrow \mathrm{FRA} \rightarrow \mathrm{FRB} \rightarrow \mathrm{FRC} \rightarrow \mathrm{FRD}$

Where FRA= Foam incorporated with Antimony trioxide/Sodium bromide $\mathrm{FRB}=$ Foam incorporated with Ammonium dihydrogen orthophosphate FRC $=$ Foam incorporated with Diammonium hydrogen orthophosphate FRD $=$ Foam incorporated with Castor oil 


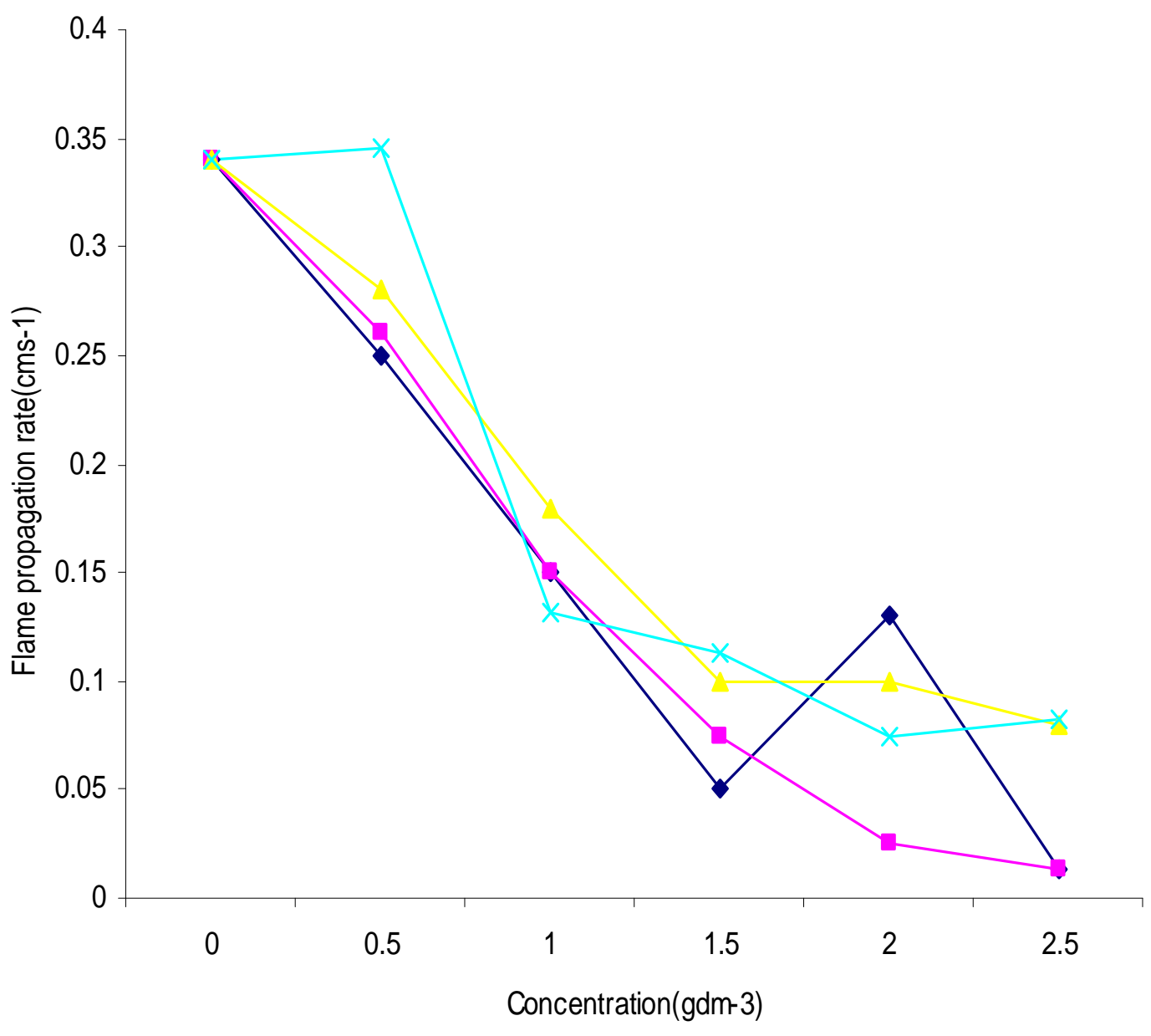

Figure 2. Effect of FR on propagation rate for flexible polyurethane foam

$$
\rightarrow \mathrm{FRA} \rightarrow-\mathrm{FRB} \longrightarrow \mathrm{FRC} \longrightarrow \mathrm{FRD}
$$

FRA $=$ Foam incorporated with Antimony trioxide/Sodium bromide $\mathrm{FRB}=$ Foam incorporated with Ammonium dihydrogen orthophosphate $\mathrm{FRC}=$ Foam incorporated with Diammonium hydrogen orthophosphate FRD $=$ Foam incorporated with Castor oil 


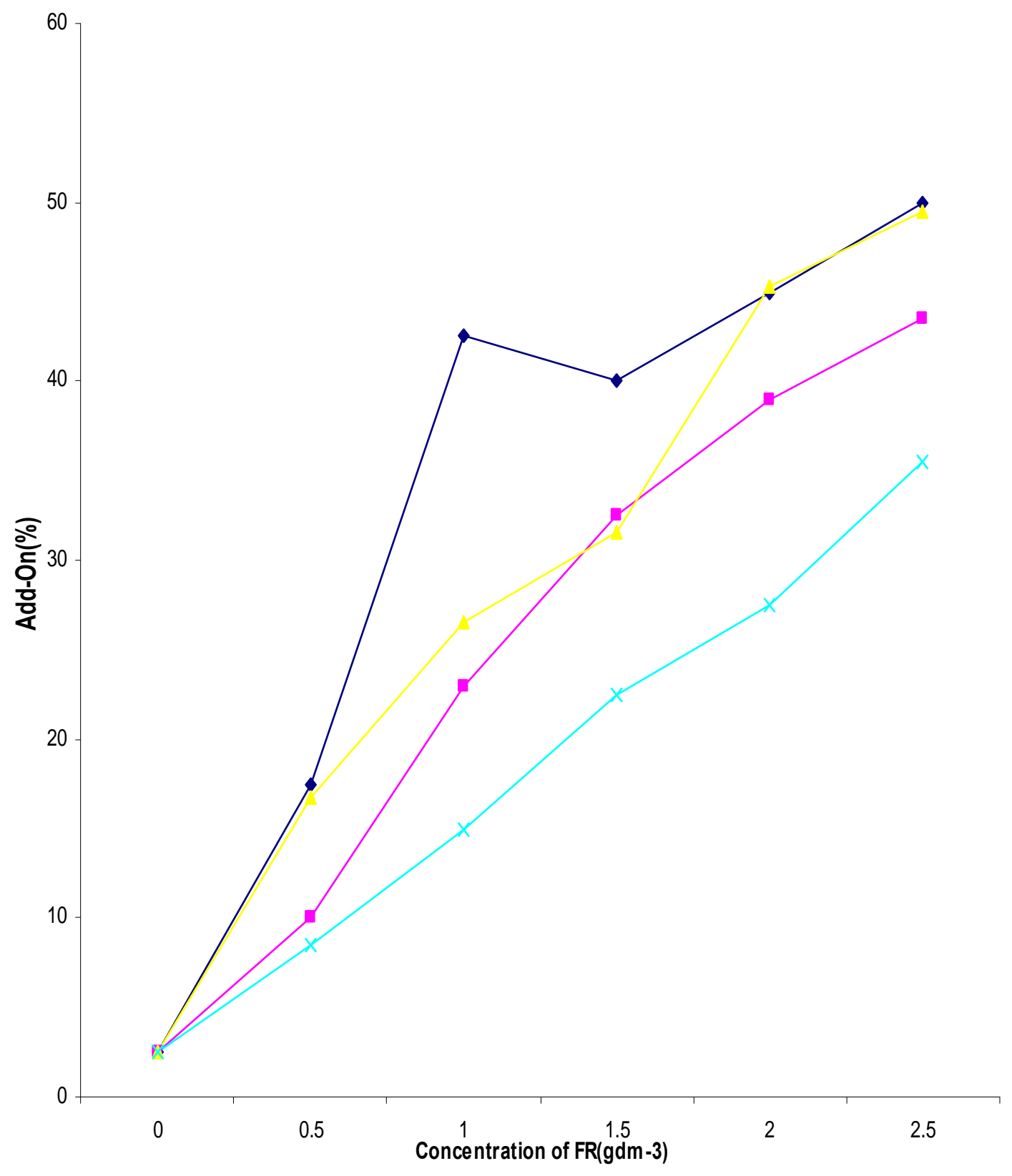

Figure 3. Effect of incorporation of FR on Add-On in flexible polyurethane foam

$$
\rightarrow \text { FRA } \leadsto \text { FRB } \_ \text {FRC } \nsim \text { FRD }
$$

FRA $=$ Foam incorporated with Antimony trioxide/Sodium bromide $\mathrm{FRB}=$ Foam incorporated with Ammonium dihydrogen orthophosphate $\mathrm{FRC}=$ Foam incorporated with Diammonium hydrogen orthophosphate $\mathrm{FRD}=$ Foam incorporated with Castor oil 


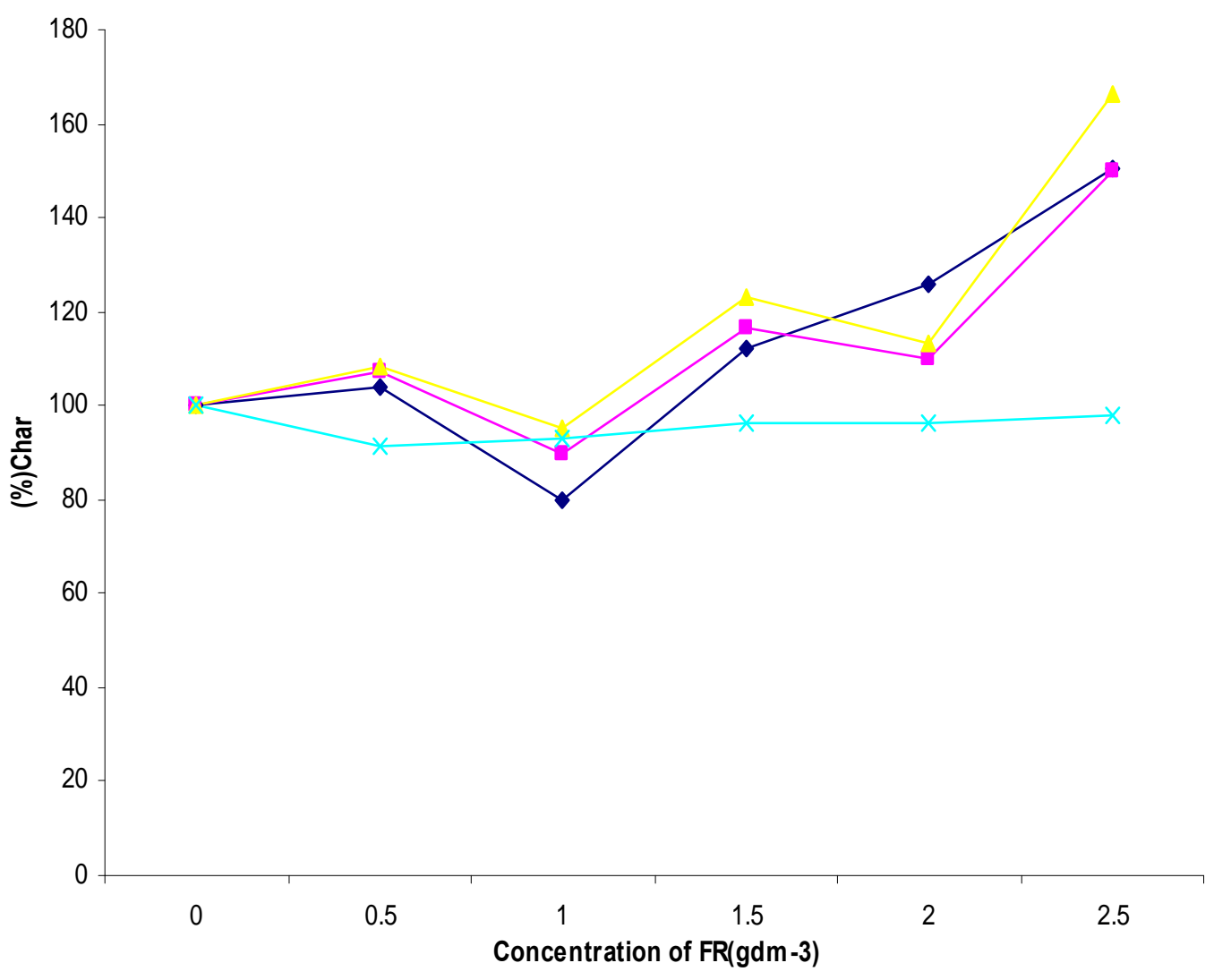

Figure 5. (\%)Char of flexible polyurethane foam versus FR concentration

$\multimap$-FRA $\rightarrow$ FRB $\rightarrow$ FRC $\quad x$ FRD

FRA $=$ Foam incorporated with Antimony trioxide/Sodium bromide $\mathrm{FRB}=$ Foam incorporated with Ammonium dihydrogen orthophosphate $\mathrm{FRC}=$ Foam incorporated with Diammonium hydrogen orthophosphate FRD $=$ Foam incorporated with Castor oil

\section{CONCLUSION}

Ordinarily polyurethane foams available in the markets are easily ignitable, highly flammable and on decomposition produce large quantity of black dense smokes containing deadly poisonous toxic gases. Impregnation of polyurethane foam with different types and concentrations of FR.increased the ignition time and the Add-On of the foam, while flame-propagation rate, After-glow, char smoke and toxicity decreased. The proportion of variations for castor showed that castor oil is a good flame retardant compared to the inorganic flame-retardants. These results confirm the added advantage of using castor oil as polyol for the foam industries. Thus by the use of flameretardants polyurethane foams in the form of mattresses, furniture cushioning and in the construction of industrial and residential buildings, life and property losses could be minimized and sufficient energy saving could be achieved.

\section{REFERENCES}

Boehling, R.S. and Cooper, J.C. (1985). Environmental fate and effects of triaryl and tri-alkyl/aryl phosphates esters. Residue Rev. 94: 49-99.

Calamari, T.A. and Harper, R.J. (1993). Flame retardants for textiles. Kirk-othman encyclopedia of chemical technology, $4^{\text {th }}$ ed. New York, John Wiley \& Sons, Vol.10,pp 998-1022.

Cullis, C.F. (1987). Bromine compounds as flame retardantsô Proceedings of the International 
conference on fire safety, Newchatal, Switzerland Vol. 12, pp. 307-323.

Gann, R.G. (1993). Flame retardants: Overview. Kirk-othman encyclopedia of chemical technology, $4^{\text {th }}$ ed. New York, John Wiley \& Sons, Vol.10,pp 930-936.

Garba, B. and Eboatu, A.N, (1990). Effect of flame retardant treatment on the thermal behaviour of some tropical timbers, J.Appl. Polym. Sci. 39; 109-112.
Herington, R. and Hock, K. (1991). Flexible Polyurethane Form. Dow Chemical Company, Midland, M.I. pp. 201.

Khattab, M.A, Kandil, S.H., Gad, A.M., Latif, E.L. and Morsi, S.E.(1992). Effects of condensed phase flame retardants on the ignition behavior of cotton fabrics. Fire Mat. 16(1): 1 